\title{
Sentinel lymph node processing in gynecological cancer histopathology and molecular biology
}

\author{
Gerlinde Lang-Avérous ${ }^{1}$, Sabrina Croce $^{2}$, Eliane Mery ${ }^{3}$, Mojgan Devouassoux-Shisheboran ${ }^{4}$ \\ ${ }^{1}$ Department of Pathology, CHU Strasbourg, Hôpital de Hautepierre, Strasbourg, France; ${ }^{2}$ Department of Pathology, Institut Bergonié, Bordeaux, \\ France; ${ }^{3}$ Department of Pathology, Institut Claudius Regaud-IUCT Oncopole, Toulouse, France; ${ }^{4}$ Department of Pathology, Hospices Civils de \\ Lyon, Université Claude Bernard Lyon I, Villeurbanne, France \\ Contributions: (I) Conception and design: All authors; (II) Administrative support: None; (III) Provision of study materials or patients: All authors; (IV) \\ Collection and assembly of data: G Lang-Avérous, M Devouassoux-Shisheboran; (V) Data analysis and interpretation: All authors; (VI) Manuscript \\ writing: All authors; (VII) Final approval of manuscript: All authors. \\ Correspondence to: Gerlinde Lang-Avérous, MD. Department of Pathology, CHU Strasbourg, Hôpital de Hautepierre, 1 Avenue Molière, 67098 \\ Strasbourg, France. Email: gerlinde.averous@chru-strasbourg.fr.
}

\begin{abstract}
As sentinel lymph nodes (SLNs) are the first nodes receiving drainage from primary tumors, they provide important prognostic information about the nodal status of a tumor. SLN biopsy has modified the lymph node assessment by pathologists. This review highlights the different ways of histopathological and molecular SLN assessment according to the different gynecological cancers. Other than in breast cancer and melanoma, frozen section (FS) analysis of SLN in gynecological malignancies is still considered an important diagnostic tool. Intraoperative evaluation of the SLN allows to determine the need of completing lymph node dissection in case of metastasis. Intraoperative FS has a high negative predictive value (NPV) and is more sensitive than imprint cytology (IC) alone. If on intraoperative examination on FS the SLN is negative, subsequent analysis of the entire lymph node and histological ultrastadification has the potential to detect occult low volume metastases or to ascertain that a SLN is really negative. This reduces the morbidity compared to systematic pelvic and paraaortic lymph node dissection. Inclusion of the entire lymph node tissue in paraffin blocks after cutting it in $2 \mathrm{~mm}$ thick slices and histopathological ultrastaging with serial sections provides important prognostic information about the need of adjuvant treatment. Three sections at 200-250 $\mu \mathrm{m}$ seem to identify the majority of micrometastases. This review discusses different histopathological protocols and molecular [qRT-PCR and one-step nucleic acid amplification $\left(\mathrm{OSNA}^{\circledR}\right)$ ] aspects of SLN evaluation in gynecological cancer.
\end{abstract}

Keywords: Sentinel lymph node (SLN); ultrastaging; metastasis; cervical cancer; endometrial cancer; vulvar cancer; vaginal cancer; intraoperative frozen section (intraoperative FS); one-step nucleic acid amplification $\left(\mathrm{OSNA}^{\circledR}\right)$

Submitted Jul 30, 2020. Accepted for publication Nov 23, 2020.

doi: 10.21037/cco-20-192

View this article at: http://dx.doi.org/10.21037/cco-20-192

\section{Introduction}

Lymph node metastasis is an important prognostic factor for gynecological cancer. Lympho-vascular invasion allows tumor cells to extend through the lymphatic system, crossing first the sentinel nodes before reaching other lymph nodes of the tumor draining lymphatic chain. Sentinel lymph nodes
(SLNs) represent the first lymph nodes to receive drainage from primary tumors. These are the lymph nodes most likely to harbour metastatic tumor cells.

Absence of tumor cells in SLNs should imply the absence of tumor spread in other lymph nodes of the lymphatic drainage system. This is why the use of standardized ultrastaging protocols for histopathological assessment of 
A

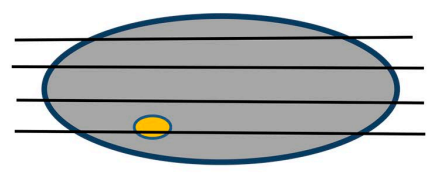

B

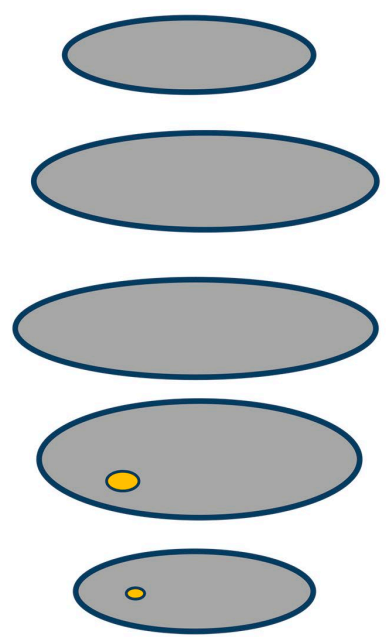

Figure 1 Macroscopic SLN procedure. (A) SLN cut in $2 \mathrm{~mm}$ slices along its long axis; (B) all slices are included in paraffin and one H\&E stained slide already allows the detection of all macrometastases (yellow cercle) $>2 \mathrm{~mm}$. SLN, sentinel lymph node; $\mathrm{H} \& \mathrm{E}$, hematoxyilin and eosin.

tumor cells in SLNs is essential.

The SLN concept was first described by Cabanas [1977] in penile cancer (1), followed by melanoma (2), breast cancer $(3,4)$, vulvar cancer $(5)$, uterine cancer $(6)$ and colon cancer (7).

The currently used TNM classification (8th edition) for gynecological cancer still considers the presence of macrometastasis (MAC) $>2 \mathrm{~mm}$ and micrometastasis (MIC) $\leq 2 \mathrm{~mm}$ as pN1 whereas isolated tumor cells (ITCs) $\leq 0.2 \mathrm{~mm}$ are considered $\mathrm{pNO} \mathrm{i}+$. In the presence of MACs and MICs in the SLN complete lymph node dissection and administration of adjuvant treatment is recommended. Whether this should also be applied to patients with ITCs is matter of debate. The diagnosis of MACs, MICs and ITCs requires meticulous histological analysis of the SLNs with ultrastaging. This is essential not only to detect metastatic tumor cells, but also to guarantee, that a SLN without tumor cells is truly negative, as patients with negative SLNs are considered of very low risk for metastasis and will not have complete pelvic lymph node dissection (PLND). The false negative rate in validation studies of pelvic sentinel nodes was less than $5 \%(8,9)$.

Gross pathological assessment of SLNs is the same, independently of tumor type and site. Some teams cut the SLNs along their long axis $(10,11)$ because the afferent lymph vessel enters the lymph node along this axis allowing more accurate detection of tumor cells in lymph node sinuses and subcapsular metastases (Figure 1). Others (12) recommend lymph node sectioning every $2 \mathrm{~mm}$ perpendicularly to its long axis in order to visualise the maximum of the lymph node surface area and subcapsular space. Both strategies require processing of the entire SLNs with histopathological ultrastaging in order to detect even very low volume metastasis (Figure 1).

Intraoperative SLN biopsy with histopathological analysis on frozen section (FS) or imprint cytology (IC) is often the first step of surgical primary management. Macroscopic examination of the SLN should look for grossly apparent MACs. A standardized sampling strategy should be applied with slicing of the SLNs at $2 \mathrm{~mm}$ intervals in order to detect MAC (smaller nodes are simply bisected). For FS ideally one or maximum two 3-5 $\mu \mathrm{m}$ thick sections are cut from each half. However, the blocks should not be deeply leveled since this may result in tissue loss for ultrastaging. For a lymph node with obvious tumor, a single FS is enough. For definitive sampling it is useful to keep the surrounding adipose tissue attached to the node in order to assess extranodal extension of the metastasis (13).

Rapid immunohistochemistry (IHC) may be necessary in case of doubt about tumor cells or mimickers such as histiocytes or dendritic cells (14), but hardly feasible in routine practice because it is time consuming.

IC is also possible, by apposition of the cutting surface of the SLN to a slide and subsequent Toluidin Blue staining. This non-expensive conservative technique reduces tissue loss and shows good specificity in the hands of a trained cytopathologist (15).

These procedures take generally 10-20 minutes allowing the surgeon to adapt surgical treatment immediately with mostly very good specificity, but rather fair sensitivity for low volume metastasis because of high false negative rates reaching up to $44 \%$ for FS (16).

\section{Intraoperative SLN assessment by molecular biology}

Using qRT-PCR, identification of specific tumor markers in lymph nodes (as cytokeratins for carcinoma cells and for exemple tyrosinase for melanoma, etc.) allows the 
detection of metastatic disease in tissue lysates. Numerous candidate genes (over 24 genes for endometrial carcinoma) with specific expression in carcinomas and no expression in lymphatic or soft tissues (17) revealed cytokeratin 19 (CK19) m-RNA as the best target to use for metastatic carcinoma cells. Togami et al. (18) used qRT-PCR to detect and quantify CK19 mRNA in endometrial carcinomas and found 104/106 (98\%) positive cases. In this study qRT-PCR of 12 pelvic lymph node (PLN) metastases reached best sensitivity and specificity without discrepancies between pathology report and molecular test when "positivity" of PLNs was determined above 4,500 copies/ $\mu \mathrm{L}$.

The one-step nucleic acid amplification $\left(\mathrm{OSNA}^{\circledR}\right)$ assay (Sysmex, Norderstedt, Germany) consists in the homogenization of lymph node tissue followed by reversetranscription loop-mediated isothermal amplification (RTLAMP) of a target mRNA, particularly CK19 mRNA when carcinoma cells are looked for. The OSNA ${ }^{\circledR}$ method consists of quantitative measurements of the target mRNA in a metastatic lymph node. Brief reaction time (30 minutes) for the entire process makes it an intraoperative tool with high specificity for the targeted mRNA in the absence of genomic DNA amplification.

In Tamaki's (19) review article, method comparison studies between $\mathrm{OSNA}^{\circledR}$ and the pathological assessment for the detection of lymph node metastasis in breast cancer revealed, that in pooled assessments OSNA ${ }^{\circledR}$ had high specificity $(94.8 \%)$, concordance $(93.8 \%)$, and negative predictive value (NPV) (97.6\%). Similar results have been found for gastric, colorectal, and lung cancers in multicenter studies (20).

SLNs are determined as 'negative' or 'positive' according to established cut-off values for the amount of CK19 mRNA-copies, related to the number of tumor cells in the sample. SLNs are classified as negative (0) for CK19 mRNA when less than $250 \mathrm{ccP} / \mu \mathrm{L}$ are detected. MICs (+) are reported when $250-5,000 \mathrm{ccP} / \mu \mathrm{L}$ and MACs (++) when $>5,000 \mathrm{mRNA} c \mathrm{P} / \mu \mathrm{L}$ are detected. SLNs containing ITCs are reported as having $<250 \mathrm{ccP} / \mathrm{mL}$. Leftover lysates could still be used for RNA extraction, determination of RNA integrity and other molecular techniques (21).

Most studies with OSNA ${ }^{\circledR}$ use the same grossing workup of SLN tissues during intraoperative assessment: after slicing the SLN in $2 \mathrm{~mm}$ thick slices, ideally alternate slices are used for classical FS or homogenized for OSNA ${ }^{\circledR}$. Small nodes $<7 \mathrm{~mm}$ are bivalved and one half used for OSNA ${ }^{\circledR}$, the other for FS and subsequent ultrastaging. After FS residual tissue is embedded and every bloc analyzed by step level sections at 200-250 $\mu \mathrm{m}$ intervals with hematoxylin and eosin (H\&E) and pan-cytokeratine stained slides (21).

As the $\mathrm{OSNA}^{\circledR}$ assay uses tissue lysates, its major drawback is the absence of morphological control of the analyzed SLN tissue. In this way, tissue allocation bias may conduct to false negative results. Another source of errors is the detection of CK19 positive benign inclusions such as endosalpingiosis, endometriosis and even mesothelial inclusions, that may generate false positive results in up to $42.3 \%$ of PLNs $(22,23)$.

Furthermore, poorly differentiated or dedifferentiated carcinomas (2\%) may be CK19 negative or express it only weakly, leading to false negative results even in the presence of MACs.

As compared to standard FS analysis or IC, the OSNA ${ }^{\circledR}$ assay is more sensitive, but also more expensive. Economic evaluations of $\mathrm{OSNA}^{\circledR}$ for the detection of SLN metastasis in breast cancer showed different results. Where GuillénParedes et al. (24) and Saruta et al. (25) found that OSNA ${ }^{\circledR}$ reduces health care costs by reducing the number of admission days and duration of surgery, Raia-Barjat et al. (26) found no difference in the expenses comparing classical FS and $\mathrm{OSNA}^{\circledR}$, but describe an important advantage of the OSNA $^{\circledR}$ avoiding another surgery for $20 \%$ of the patients. On the other hand, the systematic economic evaluation conducted by Huxley et al. 2015 (27) concludet, that OSNA is not cost-effective for the intraoperative diagnosis of SLN metastases. OSNA ${ }^{\circledR}$ is less accurate than histopathology and the consequent loss of health benefits in this patient group is not compensated by health gains elsewhere in the health system that may be obtained with the cost-savings made.

Histopathological ultrastaging consists of SLN analysis on different levels through the entire tissue bloc with combination of H\&E stained sections and IHC. Nearly all SLN tissue can be visualized this way under the microscope. Serial sections would enable analysis of the entire SLN parenchyma, but are very time consuming both for technicians and pathologists. Therefore different ultrastaging protocols have been suggested in the past in order to find the most efficient strategy $(12,13,28)$. The most ambitious ultrastaging protocols have been chosen for the evaluation of SLNs in the still ongoing trials SENTIX (NCTO2494063) and SENTICOL III (NCTO3386734) (29). Results of these trials would probably be published by the end of 2020 or 2021.

Until now, no uniform standardized histopathological work up for SLN has been adopted. Different protocols are used with varying numbers of cutting levels (3-5 and more 
if step levels through the entire bloc are chosen) and varying intervals between the slices $(40-250 \mu \mathrm{m})$. We discuss the different protocols depending on the primary tumor site below.

\section{Cervical cancer}

For two decades the SLN biopsy has become an important tool for standard care of gynecological malignancies. Apart from breast cancer, where treatment by systematic complete axillary lymph node dissection in case of intraoperatively detected SLN metastasis has been abandoned [as the ACOSOG Z0011 study (30) showed no benefit in terms of overall survival and disease free survival with systematic axillary lymph node dissection], the presence of MIC in pelvic SLNs has been linked to adverse outcomes in cervical cancer in univariate and multivariate analysis (31-33). Nowadays SLN biopsy is considered an acceptable strategy for the treatment of early stages of cervical cancer (if we exclude stage pT1a1 without lymph vessel invasion). It is recommended by the NCCN (National Comprehensive Cancer Network) guidelines, the ESGO (European Society of Gynecological Oncology), the ESTRO (Society of Radiotherapy and Oncology) and the ESP (European Society of Pathologists) as first step during surgical treatment with pelvic lymphadenectomy of other stages with the option of intraoperative assessment of the SLN.

Good SLNs biopsy performance was recently described for small tumors $<2 \mathrm{~cm}(9)$ and even for tumors between 2 and $4 \mathrm{~cm}(31,33)$.

Intraoperative assessment of SLNs is a matter of controversy since MIC and ITCs may be missed and tissue wasting may occur. In the SENTICOL trial (34) FS of SLNs had a sensitivity of only $23 \%$ and a high false negative rate for MICs and ITCs but also MACs. While the study of Roy et al. (35) showed a high false negative rate of FS for ITCs, MICs and one $2.9 \mathrm{~mm}$ MAC, Slama et al. (16) published high false negative rates of FS for MACs, MICs and ITCs with only 39 of 79 (53\%) metastases correctly detected intra-operatively. In the study of Martínez et al. (36) sensitivity and NPV of FS for the detection of macrometastatic disease was $100 \%$, sensitivity for the detection of macro and micrometastatic disease, excluding ITC, was $88.9 \%$, and NPV was $98.8 \%$. MICs and ITCs undetected on FS were found after ultrastaging in 1 patient $(1.06 \%)$ and 2 lymph nodes $(1.24 \%)$, and in 2 patients $(2.13 \%)$ and 2 lymph nodes (1.24\%), respectively. The retrospective study of Rychlik et al. (37) including FS on SLNs of 176 patients with early stage cervical cancer in two French comprehensive cancer centers showed a high NPV (97.9\%) for the detection of both macro and micrometastases.

Aditionnal FS processing would require more time and bears an important risk of artefacts and tissue loss for definitive ultrastaging. For these reasons intra-operative FS of the SLN should be avoided in cases where no complete PLND will be done (28). When we compare FS with IC, the latter is not associated with substantial tissue loss, but sensitivity is rather low (8.3-59\%) compared to FS (66\%) $(38,39)$. Slicing the lymph node in $2 \mathrm{~mm}$ sections already for intraopertive assessment increases the sensibility of IC from $56 \%$ to $75 \%$ and of FS from $60 \%$ to $100 \%(36,40)$, but augments tissue loss and is time consuming.

The intra-operative molecular detection of metastasis by OSNA $^{\circledast}$ shows a good correlation with classical SLN work-up of $96 \%(39,41)$. The study of Bizzarri et al. (42), which describes for the first time OSNA ${ }^{\circledR}$ processing of the entire lymph node tissues, detected MICs in $33.3 \%$ with a sensitivity of $85.7 \%$ and NPV of $96.1 \%$. However, we should keep in mind, that the risk of false positive results is not negligible since 19-44\% of PLNs may harbour benign CK19+ endosalpingiosis, endometriosis or mesothelial inclusions (23).

Protocoles for definitive SLN work-up are not standardized yet. Regardless of the histopathological work-up protocol, SLNs should always be cut in $2 \mathrm{~mm}$ thick slices. Several slices may be put in the same cassette. Ultrastaging is undertaken in order to enable the detection of most of the MICs and would ideally consist of analysing serial sections through the entire lymph node. However, this would be too time consuming and labour intensive for the pathologist and the laboratory staff and is not feasible in routine practice.

For this reason, different ultrastaging protocols try to approach the clinically most relevant strategy (Table 1). Euscher et al. (43) and Roy et al. (35) analysed five or six cutting levels at intervals of $40 \mu \mathrm{m}$. Lecuru et al. (29) analysed SLNs on different step sections with intervals of $200 \mu \mathrm{m}$ through the entire tissue bloc, whereas Cibula et al. (44) and Salvo et al. (9) used cutting intervals of $250 \mu \mathrm{m}$ through the entire tissue bloc. These ultrastaging protocols including H\&E staining and IHC showed increased detection rates of metastasis and relatively low false negativerates (0-8\%). At the MD Anderson Center ultrastaging used for vulvar, vaginal and cervix carcinoma consists in cutting five levels into the block at intervals of $250 \mu \mathrm{m}$ and for each 
Table 1 Histopathological ultrastaging protocols of SLN in cervical cancer

\begin{tabular}{llll}
\hline & Kocian et al. (33) [2020] & Cibula \& McCluggage (13) [2019] & Euscher \& Malpica (28) [2020] \\
\hline SLN on H\&E slide positive & No further work-up & & \\
SLN negative & Ultrastaging & & \\
& 4 levels at $150 \mu \mathrm{m}$ & 4 levels at $200 \mu \mathrm{m}$ & 5 levels at $250 \mu \mathrm{m}$ \\
& $1 \mathrm{H} \& \mathrm{E}$ and 1 unstained section per & $1 \mathrm{H} \& \mathrm{E}$ and 3 unstained sections & $1 \mathrm{H} \& \mathrm{E}$ and 2 unstained \\
& level & pertions per level & \\
H\&E slide positive & No further work-up & & \\
H\&E slide negative & Cytokeratine AE1/AE3 or cytokeratine cocktail lHC* &
\end{tabular}

*, Cytokeratine cocktail IHC includes AE1/AE3 (DAKO, 1:50, Carpinteria, CA, USA), CAM5.2 (Becton Dickinson, 1:50, San Jose, CA, USA), cytokeratin MNF116 (DAKO, 1:50, Carpinteria, CA, USA), and keratin 8 and 18 (Zymed, 1:25, South San Francisco, CA, USA) (28). SLN, sentinel lymph node; H\&E, hematoxylin and eosin; IHC, immunohistochemistry.
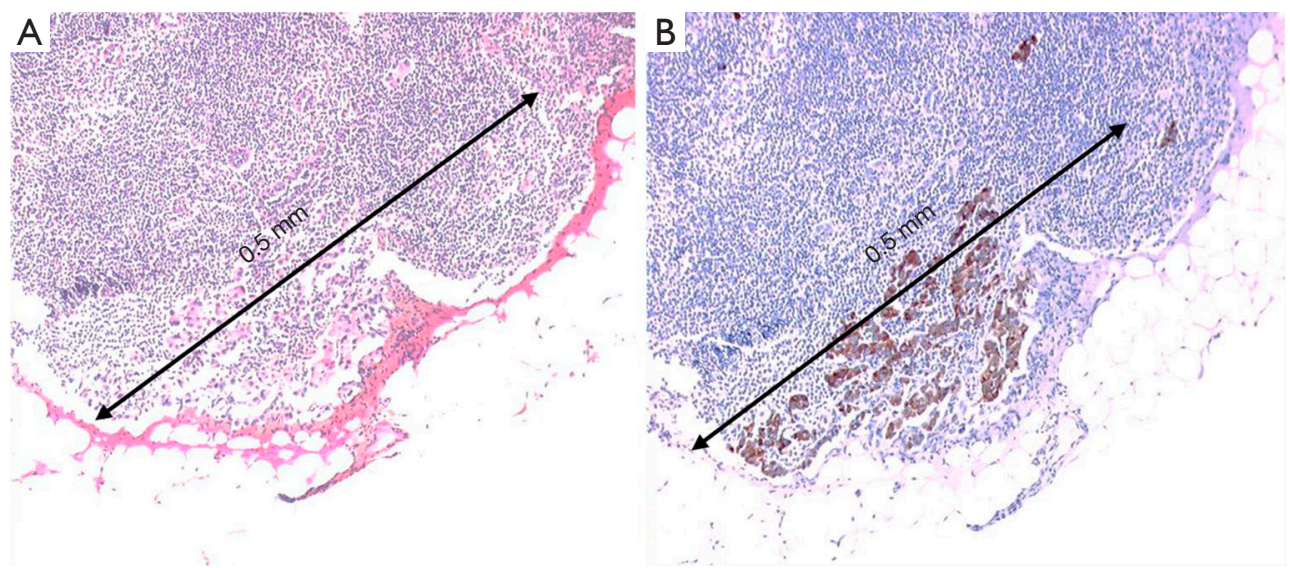

Figure 2 SLN with MIC of cervical squamous cell carcinoma $\times 100$. (A) H\&E stained SLN; (B) IHC with pan-cytokeratine AE1/AE3. MIC, micrometastasis; SLN, sentinel lymph node; H\&E, hematoxyilin and eosin; IHC, immunohistochemistry.

level 1 serial section is stained $\mathrm{H} \& \mathrm{E}$, a second is submitted for cytokeratine IHC (AE1/AE3) and a third is stored (28). At the Memorial Sloan Kettering Center NY ultrastaging is quite different and the same for cervical and endometrial cancer with two additional H\&E levels $50 \mu \mathrm{m}$ apart and two unstained slides for cytokeratin IHC (45).

In a retrospective study conducted at the Prague University Hospital (33) including 226 patients with T1a-T1b cervical cancer, treated by primary surgery and intraoperative SLN assessment by FS, ultrastaging consisted of cutting four levels into the bloc at intervals of $150 \mu \mathrm{m}$, with one $\mathrm{H} \& \mathrm{E}$ and one cytokeratine AE1/AE3 IHC.

In order to facilitate standardization Cibula and McCluggage (13) suggest cutting four levels into the bloc with intervals of $200 \mu \mathrm{m}$ and three serial sections for each level: one for $\mathrm{H} \& \mathrm{E}$ staining, one for cytokeratine IHC (mostly with the pan-cytokeratin antibody AE1/AE3) and the third one to use in case of technical problems with the first two slides or eventually to complete size evaluation of detected metastasis with $\mathrm{H} \& \mathrm{E}$ staining of the third slide.

Patients should be informed before their surgery that, despite a negative intraoperative SLN evaluation, SLN metastases may be identified later by pathological ultrastaging in $30-50 \%$ of cases (Figure 2).

\section{Endometrial cancer}

SLN mapping for endometrial cancer is not standardized yet, but is an option for the treatment of low risk endometrial carcinomas (46). A survey conducted (47) among gynecological oncologists in the United States shows that SLN mapping for endometrial cancer, especially 
Table 2 Histopathological ultrastaging protocols of SLN in endometrial cancer

\begin{tabular}{llll}
\hline & MDACC (28) & MSKCC NY (28) & SENTI-ENDO trial (53) \\
\hline SLN work-up & 1 level at $250 \mu \mathrm{m}$ & 2 levels at $50 \mu \mathrm{m}$ & 4 levels at $150 \mu \mathrm{m}$ \\
& $1 \mathrm{H} \& \mathrm{E}+2$ unstained slides & $2 \mathrm{H} \& \mathrm{E}+1$ unstained slide per level & $1 \mathrm{H \& E}+$ pan keratine IHC \\
SLN on H\&E slide positive & No further work-up & & \\
H\&E slide negative & Cytokeratine AE1/AE3 or cytokeratine cocktail IHC* & \\
\hline
\end{tabular}

*, Cytokeratine Cocktail IHC includes AE1/AE3 (DAKO, 1:50, Carpinteria, CA, USA), CAM5.2 (Becton Dickinson, 1:50, San Jose, CA, USA), Cytokeratin MNF116 (DAKO, 1:50, Carpinteria CA, USA), and Keratin 8 and 18 (Zymed, 1:25, South San Francisco, CA, USA) (28). MDACC: MD Anderson Cancer Center Houston, Houston, TX, USA; MSKCC: Memorial Sloan Kettering Cancer Center, New York, NY, USA. SLN, sentinel lymph node; H\&E, hematoxylin and eosin; IHC, immunohistochemistry.

endometrioid carcinomas grades 1 and 2, is performed by $82.7 \%$ of the members of the American Society of Gynecological Oncologists. Only 17.9\% of them do intraoperative evaluation of SLNs with FS (in half of the cases) or IC despite its deceptively low sensitivity (48).

Intraoperative SLN evaluation by $\mathrm{OSNA}^{\circledR}$ seems to be more efficient, since sensitivity and specificity are respectively $82.4-100 \%$ and $86.7-99.2 \%(17,49)$, but the technique carries the risk of false positives if benign epithelial inclusions such as endosalpingiosis or endometriosis are present in the node (10). In the study of López-Ruiz et al. (49) of 89 LNS from 34 patients 11 SLN had discordant OSNA ${ }^{\circledR}+$ /histo-results, two of them (belonging to one patient) with benign endosalpingiosis.

Fanfani et al. (21) also showed excellent accuracy (99\%) of the OSNA ${ }^{\circledR}$ assay for SLNs in endometrial cancer with a sensitivity and specificity of respectively 87.5 and $100 \%$ as well as NPV and PPV of $99 \%$ and $100 \%$. FS was less accurate $(93.6 \%)$ with low sensitivity of $50 \%$ and NPV of $14.3 \%$, while specificity and PPV of $99.4 \%$ and $99 \%$ were very good. Nevertheless, in this study one OSNA ${ }^{\circledR}$ and FS negative SLN had MAC on definitive ultrastaging. This may have occured by tissue allocation bias when using different slices for OSNA ${ }^{\circledast}$ and histopathological ultrastaging.

A retrospective study involving 396 patients with endometrial carcinoma (50) evaluated the use of histopathological ultrastaging alone (214 patients) versus OSNA $^{\circledast}$ (182 patients) showing better performance of histopathological ultrastaging alone for MACs, whereas OSNA $^{\circledR}$ performed better for MICs. OSNA ${ }^{\circledR}$ allowed upstaging of $20.69 \%$ of patients into stage FIGO III in a study with 135 SLNs out of 54 patients with endometrial carcinoma (51). Ultrastaging enhances the detection of low volume metastasis in endometrial carcinoma by up to $32 \%$ (12).
Despite NCCN guidelines (52), requiring additional pathological examination of SLNs in endometrial carcinoma with $\mathrm{H} \& \mathrm{E}$ stainings and pan keratin IHC, no recommandations are published concerning the type of ultrastaging protocol to use.

As a wide range of ultrastaging protocols are in use, it is difficult to compare the results (Table 2). The FIRES trial (54) for example, used two cutting levels at $50 \mu \mathrm{m}$ intervals with $\mathrm{H} \& \mathrm{E}$ and pan keratine IHC when the $\mathrm{H} \& \mathrm{E}$ slide was negative for tumor cells. In the French SENTIENDO study $(53,55)$ SLN ultrastaging consisted in cutting four levels at intervals of $150 \mu \mathrm{m}$ into the bloc with $\mathrm{H} \& \mathrm{E}$ staining and pan keratine IHC for each level (43) compared two different strategies, one using five cutting levels at intervals of $250 \mu \mathrm{m}$ with at each level H\&E staining and IHC versus one HE level and one IHC at $250 \mu \mathrm{m}$. There was no significant difference between the two protocols, and $70 \%$ of the metastasis showed up on the first level. The recently published review article by Euscher and Malpica (28) listed different ultrastaging protocols with varying numbers of cutting levels (1-6 mostly 4) at different intervals (40-200 $\mu \mathrm{m})$ and showed no significant differences in both the overall rate of detected metastasis (12.6-30\%), and the number of patients with false negative SLNs [0-5]. Upstaging due to ultrastaging occured in 10-44\% (Figure 3).

Kennard et al. (56) studied the relationship between primary tumor histology, SLN metastasis, and non-sentinel node metastasis in endometrial cancer of 275 low risk (LR), 80 intermediate risk (IR) and 59 high risk (HR) carcinomas. They found important correlations between the presence of ITCs in SLNs and the presence of lymph node metastasis in pelvic non SLN $(\mathrm{P}=0.03)$ and paraortic nodes $(\mathrm{P}=0.008)$ for all histological sub-groups. ITCs were more frequently present in LR and IR cancers (51.6\% and $44.7 \%$ ) compared to HR 

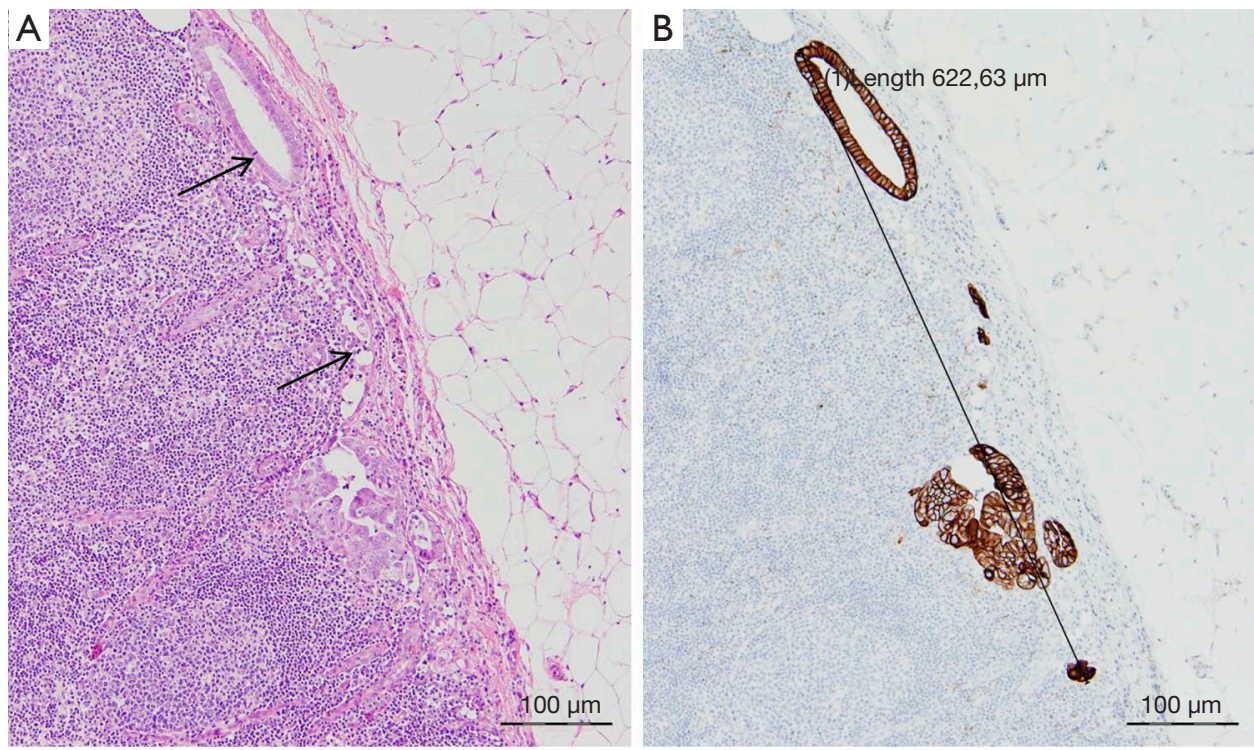

Figure 3 SLN with MIC of endometrioid adenocarcinoma $\times 100$. (A) H\&E stained SLN. Arrows indicate MIC; (B) pan-cytokeratin AE1/ AE3 IHC. SLN, sentinel lymph node; MIC, micrometastasis; H\&E, hematoxyilin and eosin; IHC, immunohistochemistry.

cancers (15\%). When ITCs were present in the SLN, 8\% of PLNs showed metastasis, versus 31.5\% when SLNs had MAC or MICs. This study identifies a significant association between pelvic and para-aortic lymph node metastasis. Paraaortic lymph nodes had metastasis in $33 \%$ of HR tumors with ITCs in the SLN. Furthermore in HR cancers (serous, clear cell, undifferentiated, carcinosarcoma and grade 3 endometrioid carcinomas), positive SLNs have an increased risk of metastasis in non-SLN (37\%), while LR cancers show negative non SLNs in over $75 \%$ of the cases (43).

The Canadian study (57) including 517 patients with endometrial cancer showed SLN metastasis in 85 (16.4\%) patients of which 31 (36\%) ITCs. Of these 31 patients with ITCs in the SLN, 11 patients were treated by chemotherapy and whole pelvis radiation therapy (WPRT), 10 patients with WPRT alone and 10 patients had no adjuvant treatment or only local vault brachytherapy (VBT). Only one relaps was noted but it occured in a patient with carcinosarcoma. Progression free survival (PFS) at 3-year for patients with ITCs in the SLN was $95.5 \%$, almost identical to node negative patients (87.6\%) and those with MIC (85.5\%). No tumor with endometrioid histology recurred $(0 / 28)$ during this follow-up period.

However, to this day the role of ITCs in endometrial carcinoma remains still unclear. Pathologists should report size of metastatic foci. Like in breast cancer, the American Joint Commitée of Cancer (AJCC) has applied the size of lymph node metastasis to the tumor classification items: $>2 \mathrm{~mm}=$ MAC, $>0.2-2 \mathrm{~mm}=\mathrm{MIC}$ and $<0.2 \mathrm{~mm}=$ ITC and has recently considered ITCs as pNOi+ without upstaging the patient. It remains to date unclear as to whether this should be applied to all types of endometrial carcinoma.

\section{Vulvar cancer}

Vulva was the first gyneocological site studied for SLN mapping due to its superficial location and easily predictable lymphatic drainage. Multiple studies have shown that the technique was able to give accurate information about prognosis, and allowed to adapt individual treatment and reduced morbidity. Since the GROningen International Study on Sentinel Nodes in Vulvar cancer (GROINSS-V) (58) as well as the 173 GOG study (8), it has become a standard of care for vulvar invasive squamous cell carcinomas for patients with stage $\mathrm{T} 1$ and $\mathrm{T} 2<4 \mathrm{~cm}$ unifocal tumors, with negative clinical groin examination, negative imaging and no previous vulvar surgery, that could have altered lymphatic drainage. Ultrastaging of SLNs is recommended but to date, no standardized protocol has been adopted. In studies published between 2007 and 2018 the number of cutting levels varies from 1 to 7 , at intervals of 40 to $1,000 \mu \mathrm{m}$ with most of them using pan keratine IHC. False negative rates were low $(0-2.4 \%)$, with metastases detected in $17-33 \%$ of patients with ultrastaging and 
upstaging in $8.6 \%$ to $41.7 \%$ of the cases (28). All studies emphasize the importance of ultrastaging for SLNs because undetected metastases in a SLN carry an important risk of groin recurrence (59). Although the rate of positive non sentinel nodes was relatively low $(4.25 \%)$ for ITCs in the SLN (GROINSS-V study), experts agreed, that all patients with positive SLNs in vulvar cancer would need additional treatment including those with MICs and ITCs.

\section{Vaginal cancer}

Vaginal carcinoma is very rare and concerns mostly squamous cell carcinomas (95\%) usually diagnosed at an advanced age. Very few reported studies concern SLN mapping especially for squamous carcinoma or melanoma with regards towards technical aspects of the mapping procedure. They describe the detection of SLN mostly in the groin or in the pelvis without detailed pathology reporting. The German Cancer Society (DKG), the Gynecologic Oncology Working Group (AGO) and the German Society of Gynecology and Obstetrics (DGGG) recommend the detection of SLN in vaginal cancer using ultrastaging and IHC (60) but mention that to date the evidence of MIC is not relevant for the staging of vaginal cancer. To date no standardized ultrastaging protocol is available. Yet, the importance of SLN mapping was emphasized, as drainage by lymphatic channels does not always follow the anatomically predicted pathways. Localization of the SLN and lymph drainage pathways represent important information for the radiotherapist (61).

\section{Conclusions}

There is a need for standardization of histological ultrastaging procedures in order to better evaluate the impact of SLN diagnosis.

Detailed ultrastaging for detection of ITCs and MIC is crucial until randomized trials proove oncological safety of more lenient protocols.

Molecular SLN analysis is an intraoperative very sensitive and quick procedure, but it is more expensive than microscopic evaluation and carries the risk of false positives, especially in patients with pelvic endometriosis or endosalpingiosis.

\section{Acknowledgments}

Funding: None.

\section{Footnote}

Provenance and Peer Review: This article was commissioned by the Guest Editors (Denis Querleu and Cherif Youssef Akladios) for the series "Sentinel Lymph Node Biopsy in Gynecologic Cancer" published in Chinese Clinical Oncology. The article was sent for external peer review organized by the Guest Editors and the editorial office.

Conflicts of Interest: All authors have completed the ICMJE uniform disclosure form (available at http://dx.doi. org/10.21037/cco-20-192). The series "Sentinel Lymph Node Biopsy in Gynecologic Cancer" was commissioned by the editorial office without any funding or sponsorship. The authors have no other conflicts of interest to declare.

Ethical Statement: The authors are accountable for all aspects of the work in ensuring that questions related to the accuracy or integrity of any part of the work are appropriately investigated and resolved.

Open Access Statement: This is an Open Access article distributed in accordance with the Creative Commons Attribution-NonCommercial-NoDerivs 4.0 International License (CC BY-NC-ND 4.0), which permits the noncommercial replication and distribution of the article with the strict proviso that no changes or edits are made and the original work is properly cited (including links to both the formal publication through the relevant DOI and the license). See: https://creativecommons.org/licenses/by-nc-nd/4.0/.

\section{References}

1. Cabanas RM. An approach for the treatment of penile carcinoma. Cancer 1977;39:456-66.

2. Morton DL, Wen DR, Wong JH, et al. Technical details of intraoperative lymphatic mapping for early stage melanoma. Arch Surg 1992;127:392-9.

3. Krag DN, Weaver DL, Alex JC, et al. Surgical resection and radiolocalization of the sentinel lymph node in breast cancer using a gamma probe. Surg Oncol 1993;2:335-9; discussion 340.

4. Giuliano AE, Kirgan DM, Guenther JM, et al. Lymphatic mapping and sentinel lymphadenectomy for breast cancer. Ann Surg 1994;220:391-8.

5. de Hullu JA, Doting E, Piers DA, et al. Sentinel lymph node identification with technetium-99m-labeled nanocolloid in squamous cell cancer of the vulva. J Nucl 
Med 1998;39:1381-5.

6. Huchon C, Bats AS, Achouri A, et al. Sentinel lymph node procedure and uterine cancers. Gynecol Obstet Fertil 2010;38:760-6.

7. Saha S, Elgamal M, Cherry M, et al. Challenging the conventional treatment of colon cancer by sentinel lymph node mapping and its role of detecting micrometastases for adjuvant chemotherapy. Clin Exp Metastasis 2018;35:463-9.

8. Levenback CF, Ali S, Coleman RL, et al. Lymphatic mapping and sentinel lymph node biopsy in women with squamous cell carcinoma of the vulva: a gynecologic oncology group study. J Clin Oncol 2012;30:3786-91.

9. Salvo G, Ramirez PT, Levenback CF, et al. Sensitivity and negative predictive value for sentinel lymph node biopsy in women with early-stage cervical cancer. Gynecol Oncol 2017;145:96-101.

10. Alsadoun N, Devouassoux-Shisheboran M. Pathological process for sentinel lymph node. Bull Cancer 2020;107:642-52.

11. Weaver DL. Pathology evaluation of sentinel lymph nodes in breast cancer: protocol recommendations and rationale. Mod Pathol 2010;23 Suppl 2:S26-32.

12. Euscher E, Sui D, Soliman P, et al. Ultrastaging of sentinel lymph nodes in endometrial carcinoma according to use of 2 different methods. Int J Gynecol Pathol 2018;37:242-51.

13. Cibula D, McCluggage WG. Sentinel lymph node (SLN) concept in cervical cancer: Current limitations and unanswered questions. Gynecol Oncol 2019;152:202-7.

14. Krishnamurthy S, Meric-Bernstam F, Lucci A, et al. A prospective study comparing touch imprint cytology, frozen section analysis, and rapid cytokeratin immunostain for intraoperative evaluation of axillary sentinel lymph nodes in breast cancer. Cancer 2009;115:1555-62.

15. Pétursson HI, Kovács A, Mattsson J, et al. Evaluation of intraoperative touch imprint cytology on axillary sentinel lymph nodes in invasive breast carcinomas, a retrospective study of 1227 patients comparing sensitivity in the different tumor subtypes. PloS One 2018;13:e0195560.

16. Slama J, Dundr P, Dusek L, et al. High false negative rate of frozen section examination of sentinel lymph nodes in patients with cervical cancer. Gynecol Oncol 2013;129:384-8.

17. Nagai T, Niikura H, Okamoto S, et al. A new diagnostic method for rapid detection of lymph node metastases using a one-step nucleic acid amplification (OSNA) assay in endometrial cancer. Ann Surg Oncol 2015;22:980-6.

18. Togami S, Kawamura T, Fukuda M, et al. Quantitative
RT-PCR assay for detecting lymph node metastasis in endometrial cancer: a preliminary study. Oncology 2019;96:179-82.

19. Tamaki Y. One-step nucleic acid amplification (OSNA): where do we go with it? Int J Clin Oncol 2017;22:3-10.

20. Shoji Y, Kumagai K, Kamiya S, et al. Prospective feasibility study for single-tracer sentinel node mapping by ICG (indocyanine green) fluorescence and OSNA (one-step nucleic acid amplification) assay in laparoscopic gastric cancer surgery. Gastric Cancer 2019;22:873-80.

21. Fanfani F, Monterossi G, Ghizzoni V, et al. One-Step Nucleic Acid Amplification (OSNA): A fast molecular test based on CK19 mRNA concentration for assessment of lymph-nodes metastases in early stage endometrial cancer. PLoS One 2018;13:e0195877.

22. Tempfer CB, Wenzl R, Horvat R, et al. Lymphatic spread of endometriosis to pelvic sentinel lymph nodes: a prospective clinical study. Fertil Steril 2011;96:692-6.

23. Noël JC, Chapron C, Fayt I, et al. Lymph node involvement and lymphovascular invasion in deep infiltrating rectosigmoid endometriosis. Fertil Steril 2008;89:1069-72.

24. Guillén-Paredes MP, Carrasco-González L, et al. Onestep nucleic acid amplification (OSNA) assay for sentinel lymph node metastases as an alternative to conventional postoperative histology in breast cancer: a cost-benefit analysis. Cirugia Espanola 2011;89:456-62.

25. Saruta Y, Puig-Junoy J. Cost and Budget Impact Analysis of an Accurate Intraoperative Sentinel Lymph Node Diagnosis for Breast Cancer Metastasis. Appl Health Econ Health Policy 2016;14:323-35.

26. Raia-Barjat T, Trombert B, Khaddage A, et al. OSNA (one-step nucleic acid amplification) sentinel lymph node intraoperative molecular analysis in breast cancer: a costbenefit analysis. Med Oncol 2014;31:322.

27. Huxley N, Jones-Hughes T, Coelho H, et al. A systematic review and economic evaluation of intraoperative tests RD-100i one-step nucleic acid amplification (OSNA) system and Metasin test for detecting sentinel lymph node metastases in breast cancer. Health Technol Assess 2015;19:v-xxv, 1-215.

28. Euscher ED, Malpica A. Gynaecological malignancies and sentinel lymph node mapping: an update. Histopathology 2020;76:139-50.

29. Lecuru FR, McCormack M, Hillemanns P, et al. SENTICOL III: an international validation study of sentinel node biopsy in early cervical cancer. A GINECO, ENGOT, GCIG and multicenter study. Int J Gynecol 


\section{Page 10 of 11}

Cancer 2019;29:829-34.

30. Jorns JM, Kidwell KM. Sentinel lymph node frozensection utilization declines after publication of american college of surgeons oncology group z0011 trial results with no change in subsequent surgery for axillary lymph node dissection. Am J Clin Pathol 2016;146:57-66.

31. Dostalek L, Åvall-Lundqvist E, Creutzberg CL, et al. ESGO Survey on Current Practice in the Management of Cervical Cancer. Int J Gynecol Cancer 2018;28:1226-31.

32. Guani B, Dorez M, Magaud L, et al. Impact of micrometastasis or isolated tumor cells on recurrence and survival in patients with early cervical cancer: SENTICOL Trial. Int J Gynecol Cancer 2019;29:447-52.

33. Kocian R, Slama J, Fischerova D, et al. Micrometastases in sentinel lymph nodes represent a significant negative prognostic factor in early-stage cervical cancer: a singleinstitutional retrospective cohort study. Cancers (Basel) 2020;12:1438.

34. Bats AS, Mathevet P, Buenerd A, et al. The sentinel node technique detects unexpected drainage pathways and allows nodal ultrastaging in early cervical cancer: insights from the multicenter prospective SENTICOL study. Ann Surg Oncol 2013;20:413-22.

35. Roy M, Bouchard-Fortier G, Popa I, et al. Value of sentinel node mapping in cancer of the cervix. Gynecol Oncol 2011;122:269-74.

36. Martínez A, Mery E, Filleron T, et al. Accuracy of intraoperative pathological examination of SLN in cervical cancer. Gynecol Oncol 2013;130:525-9.

37. Rychlik A, Angeles MA, Migliorelli F et al. Frozen section examination of sentinel lymph nodes can be used as a decisional tool in the surgical management of early cervical cancer. Int J Gynecol Cancer 2020;30:358-63.

38. Sonoda K, Yahata H, Okugawa K, et al. Value of intraoperative cytological and pathological sentinel lymph node diagnosis in fertility-sparing trachelectomy for earlystage cervical cancer. Oncology 2018;94:92-8.

39. Barranger E, Bricou A, Morel O, et al. Biopsie du ganglion sentinelle dans le cancer du col de l'utérus: état de l'art en 2007. Gynécologie Obstétrique Fertil 2007;35:516-22.

40. Niikura H, Okamoto S, Otsuki T, et al. Prospective study of sentinel lymph node biopsy without further pelvic lymphadenectomy in patients with sentinel lymph node-negative cervical cancer. Int J Gynecol Cancer 2012;22:1244-50.

41. Okamoto S, Niikura H, Nakabayashi K, et al. Detection of sentinel lymph node metastases in cervical cancer: assessment of KRT19 mRNA in the one-step nucleic

\section{Lang-Avérous et al. SLN processing in gynecological cancer}

acid amplification (OSNA) method. Gynecol Oncol 2013;130:530-6.

42. Bizzarri N, Anchora LP, Zannoni GF, et al. Role of onestep nucleic acid amplification (OSNA) to detect sentinel lymph node low-volume metastasis in early-stage cervical cancer. Int J Gynecol Cancer 2020;30:364-71.

43. Euscher ED, Malpica A, Atkinson EN, et al. Ultrastaging improves detection of metastases in sentinel lymph nodes of uterine cervix squamous cell carcinoma. Am J Surg Pathol 2008;32:1336-43.

44. Cibula D, Pötter R, Planchamp F, et al. The European Society of Gynaecological Oncology/European Society for Radiotherapy and Oncology/European Society of Pathology Guidelines for the management of patients with cervical cancer. Int J Gynecol Cancer 2018;28:641-55.

45. Diaz JP, Gemignani ML, Pandit-Taskar N, et al. Sentinel lymph node biopsy in the management of early-stage cervical carcinoma. Gynecol Oncol 2011;120:347-52.

46. Ballester M, Dubernard G, Lécuru F, et al. Detection rate and diagnostic accuracy of sentinel-node biopsy in early stage endometrial cancer: a prospective multicentre study (SENTI-ENDO). Lancet Oncol 2011;12:469-76.

47. Renz M, Diver E, English D, et al. Sentinel lymph node biopsies in endometrial cancer: practice patterns among gynecologic oncologists in the United States. J Minim Invasive Gynecol 2020;27:482-8.

48. Delpech Y, Cortez A, Coutant C, et al. The sentinel node concept in endometrial cancer: histopathologic validation by serial section and immunohistochemistry. Ann Oncol 2007;18:1799-803.

49. López-Ruiz ME, Diestro MD, Yébenes L, et al. Onestep nucleic acid amplification (OSNA) for the detection of sentinel lymph node metastasis in endometrial cancer. Gynecol Oncol 2016;143:54-9.

50. Fanfani F, Monterossi G, Meo MLD, et al. Standard ultrastaging compared to one-step nucleic acid amplification for the detection of sentinel lymph node metastasis in endometrial cancer patients: a retrospective cohort comparison. Int J Gynecol Cancer 2020;30:372-7.

51. Kost'un J, Pešta M, Sláma J, et al. One-step nucleic acid amplification vs ultrastaging in the detection of sentinel lymph node metastasis in endometrial cancer patients. J Surg Oncol 2019:119:361-9.

52. Holloway RW, Abu-Rustum NR, Backes FJ, et al. Sentinel lymph node mapping and staging in endometrial cancer: A Society of Gynecologic Oncology Literature Review with Consensus Recommendations. Gynecol Oncol 2017;146:405-15. 
53. Daraï E, Dubernard G, Bats AS, et al. Sentinel node biopsy for the management of early stage endometrial cancer: long-term results of the SENTI-ENDO study. Gynecol Oncol 2015;136:54-9.

54. Rossi EC, Kowalski LD, Scalici J, et al. A comparison of sentinel lymph node biopsy to lymphadenectomy for endometrial cancer staging (FIRES trial): a multicentre, prospective, cohort study. Lancet Oncol 2017;18:384-92.

55. Ballester M, Dubernard G, Bats AS, et al. Comparison of diagnostic accuracy of frozen section with imprint cytology for intraoperative examination of sentinel lymph node in early-stage endometrial cancer: results of Senti-Endo study. Ann Surg Oncol 2012;19:3515-21.

56. Kennard JA, Stephens AJ, Ahmad S, et al. Sentinel lymph nodes (SLN) in endometrial cancer: the relationship between primary tumor histology, SLN metastasis size, and non-sentinel node metastasis. Gynecol Oncol 2019;154:53-9.

Cite this article as: Lang-Avérous G, Croce S, Mery E, Devouassoux-Shisheboran M. Sentinel lymph node processing in gynecological cancer histopathology and molecular biology. Chin Clin Oncol 2021;10(2):17. doi: 10.21037/cco-20-192
57. Plante M, Stanleigh J, Renaud MC, et al. Isolated tumor cells identified by sentinel lymph node mapping in endometrial cancer: does adjuvant treatment matter? Gynecol Oncol 2017;146:240-6.

58. Van der Zee AGJ, Oonk MH, De Hullu JA, et al. Sentinel node dissection is safe in the treatment of early-stage vulvar cancer. J Clin Oncol 2008;26:884-9.

59. Sykes P, Eva L, van der Griend R, et al. Pathological process has a crucial role in sentinel node biopsy for vulvar cancer. Gynecol Oncol 2019;153:292-6.

60. Schnürch HG, Ackermann S, Alt-Radtke CD, et al. Diagnosis, therapy and follow-up of vaginal cancer and its precursors. Guideline of the DGGG and the DKG (S2kLevel, AWMF Registry No.032/042, October 2018). Geburtshilfe Frauenheilkd 2019;79:1060-78.

61. Frumovitz M, Gayed IW, Jhingran A, et al. Lymphatic mapping and sentinel lymph node detection in women with vaginal cancer. Gynecol Oncol 2008;108:478-81. 